\title{
Matrix of pairwise comparison: decision making with the analytic network process
}

\author{
Aliya Akhmadullina ${ }^{1}$,Svetlana Vasilyeva $^{2}$, Tatyana Yakovleva $^{2}$, Svetlana Vopiyashina $^{2}$, \\ Raisa $\mathrm{Kraineva}^{3,{ }^{*}}$ \\ ${ }^{1}$ Kazan Federal University, Institute of Management, Economics and Finance, 420008, Kazan, Russia \\ ${ }^{2}$ Togliatti State University, Institute of Finance, Economics and Management, Belorusskaya Ulitsa, \\ 14, Tolyatti, Samarskaya oblast', 445020 Russia \\ ${ }^{3}$ Volga Region State University of Service, Institute of Economics, Ulitsa Gagarina, 4, Tolyatti, \\ Samarskaya oblast', 445017, Russia
}

\begin{abstract}
This article describes a method for analyzing hierarchies; identifies the problems with inconsistent judgments. The proof is given that the most effective tool allowing one to make the right decisions with inconsistencies is the introduction of the eigenvector on environmental planning and management. The Analytic Hierarchy Process (AHP) is a method for decision making, which includes qualitative factors. In this method, ratio scales are obtained from ordinal scales, which are derived from individual judgments for qualitative factors using the pairwise comparison matrix. This paper describes the applicability of a multicriteria decisionmaking method, specifically, the analytic network process.
\end{abstract}

\section{Statement of the problem}

The economic theory of the multidimensional stock and goods market considered in this work belongs to positive sciences as it describes what is, not what should be, which is characteristic areas of research of standard sciences. As shown in work, positive sciences essentially abstain from moral and ideological judgments concerning that, properly to treat the described phenomena, and limit themselves to studying of regularities of the studied objects. Though in practice it is never possible to draw an accurate distinction between positivistic and standard approaches, the difference between them is obvious [1].

The analysis, synthesis, induction and deduction belong to traditional methodological approaches of attracting green investment. In the theory of demand (or supply) as the analysis we will understand mental decomposition of volume of demand (supply) for parts with studying of each part determined by the corresponding factor of demand (supply). And under synthesis - connection of separate parts of demand (supply) in whole [2].

As for induction, it is one of the main methods of scientific knowledge demanding collecting and studying of specific factors and generalization of the received conclusions [3]. If induction is the movement "from the particular to the general", deduction - "from the general to the particular".

\footnotetext{
* Corresponding author: Ajdar.Ajupov@kpfu.ru
} 
It is possible to refer the principles of a determinism to number of the basic methodological principles of the economic theory of the market, an indeterminism, the principle of "Okkam's razor" and others.

For each good in each country, there are structural forms of the competitive market [4]. Carrying out in-depth economic system studies in the sphere of the competitive markets needs new approaches.

The basis of this approach, in our view, are the fundamental principles of research systems. They include principles of a determinism and indeterminism formulated for the first time respectively by Pierre Laplace (1749-1827) and Leon Brilluen (1889-1969) for research of physical systems and processes. Relying on methodologies of the economic theory and general theory of systems and considering the competitive markets from system positions, we will formulate the basic principles of research and the competitive markets [5].

Today, due to the multiple complexity of society, the decision-making process urgently requires comprehensive scientific methodology on environmental planning and management.

While making management decisions and predict outcomes in practice people usually face a large number of different components. One of the effective tools allowing one to make a choice and forecast is the method of pair wise comparisons and simulation.

Simulation as a researching method emerged in the early sixties of XX century, because it was most powerful and flexible technique of research and design of complex systems. Usually a simulation model is understood as a set of activities and programs for the imitation of the operation of a real-world process or system over time. It solves the task of describing the functions of sub-systems and components, the interaction between them. Such model represents the key characteristics, behaviors and functions of the real system and allows you to research, draw conclusions and make the necessary changes, eliminating undesirable properties of the system $[1-7,11]$.

Table 1. Schemes of Classification of types managerial decisions.

\begin{tabular}{|c|l|l|}
\hline № & Classification signs of types of decisions & \multicolumn{1}{c|}{ Types of decisions } \\
\hline 1 & The conditions of implementation & Routine, unique, creative \\
\hline 2 & The degree of uncertainty & Decisions under certainty, under risk \\
\hline 3 & The number of selection criteria & A single-criterion, multi-criteria \\
\hline 4 & The use of information & Deterministic, probabilistic \\
\hline 5 & Used tools & Forecasting, modeling, analysis, synthesis \\
\hline
\end{tabular}

Visual simulation system allows simulate the behavior of a discrete non-deterministic production system of the shipment without making manual explicitly numerous recurrence equation, representing the balance equation in the mathematical model, but "assumes" compilation of such equations and their computer implementation based on structure model with multiple feedbacks and delay lines. Visual simulation computer system eliminates the need of explicit "making formulas" that is it automatically make the equation while the structural and functional models are designed in a visual mode of ready-made blocks, which area "hiding" computer program modules, those implements a certain system of equations in accordance with the purpose and settings of blocks) (example Fig.1, 2) [8-10, 12-18]. 


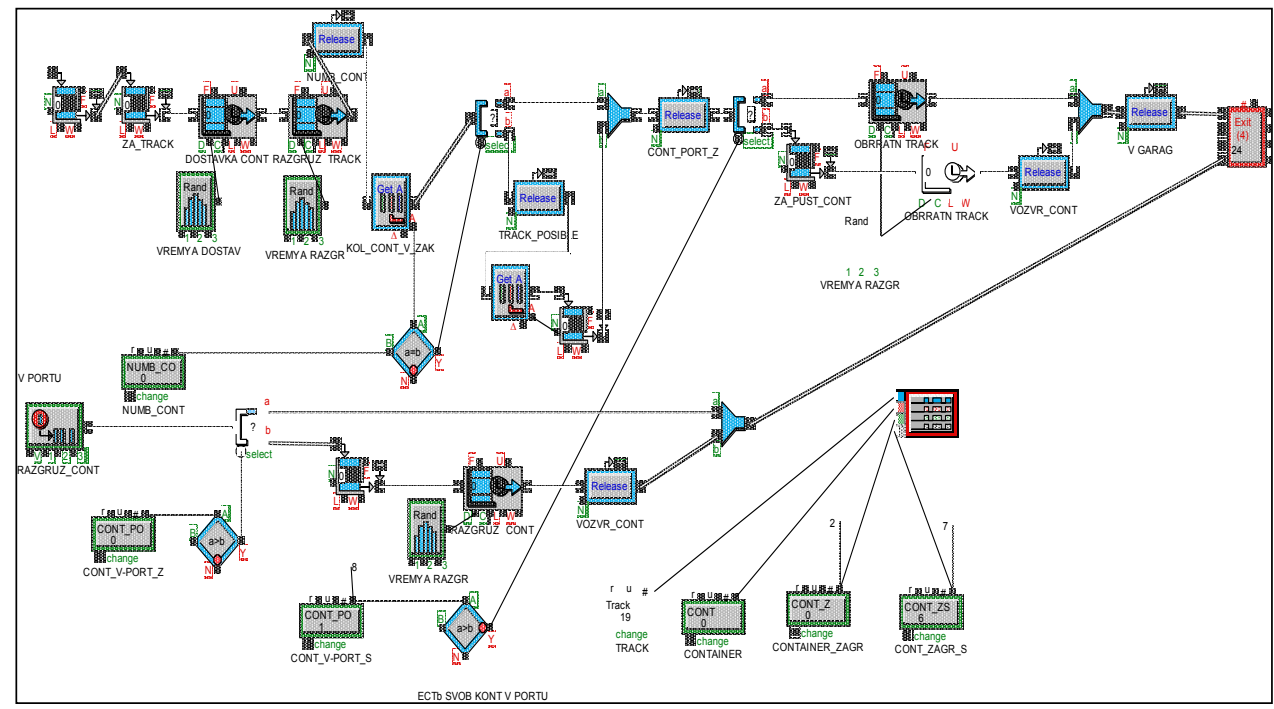

Fig. 1. Example simulation of the unloading of transport in the river port.

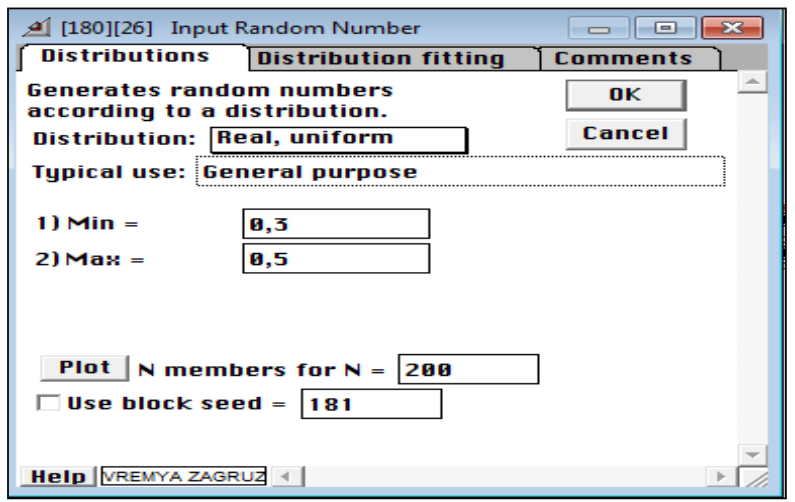

Fig. 2. Example description of the probability distribution of load time in dialog box of the block Input Random Number.

But the traditional use of this method does not answer the question of what to do if judgment is not consistent.

The different proposals are put forward in many works of foreign and domestic scholars. In our opinion, the most effective proposal is the introduction of the eigenvector.

The main eigenvector is a necessary representation of the priorities obtained on the basis of positive anti-symmetric matrix of pairwise comparisons:

$$
\mathrm{A}=\left(\mathrm{a}_{\mathrm{ij}}\right) \text {, }
$$

when $\mathrm{A}$ is a small perturbation of a coherent matrix.

\section{The purpose and methods of research}

In the formulation of numerical judgments, the individual tries to estimate consistently the main scale of the relationship or its equivalent in the form of a coherent matrix of relations. Approximation to coherent matrix is important when we are talking about uncertainty. 
Human judgment is necessarily inconsistent, and, if it is possible to reduce inconsistency by means of the new information, it can improve the validity of the decision. Judgment is much more sensitive to large deviations than to small ones, and therefore, once it reached a position about consistency, it becomes uncertain which coefficients should be slightly modified in order to transform the matrix of "almost coherent" into "coherent". If these changes are too strong they will make outrage and break the validity of the obtained weight vector representing the main decision.

In the field of decision-making, the concept of priority is extremely important, and therefore the procedure of removing priorities affects the performed choice.

Priorities must be unique and not variable, they should also reflect the order of preference expressed by the matrix of pairwise comparisons. The idea of the vector of priorities has a much smaller meaning to an arbitrary anti-symmetric matrix than to coherent matrix or almost coherent $A=\left(a_{i j}\right)[19$ - 22].

Positive matrix of order $n$ by $n$ is anti-symmetric if $a_{\mathrm{ji}}=.1 / a_{\mathrm{ij}}$. She is coherent, if

$$
a_{i j} a_{j k}=a_{i k} ; \quad i ; j ; k=1 ; \ldots ; n
$$

From aij =aik/ajk we have that $\mathrm{a}_{\mathrm{ji}}=\mathrm{a}_{\mathrm{jk}} / \mathrm{a}_{\mathrm{ik}}=\mathrm{a}_{\mathrm{ij}}{ }^{-1}$ and, consequently, a coherent matrix is ant symmetric.

It is considered that the matrix is almost consensual, if it is a little different from the consensual. Usually one looks for the vector $\mathrm{w}=(. \mathrm{w} 1 ; \ldots$; wn $)$ such as that the matrix $\mathrm{W}=\{. \mathrm{wi} / \mathrm{wj}\}$ is "close" to $\mathrm{A}=($ aij $)$ in the sense of minimizing the metric. The proximity metric to the numerical values of the aij says little about the numerical precision with which one element is directly superior to another in the matrix and it seems to be degrees of the matrix indirectly through the other elements.

\section{The results of the research}

According to the theory of Saaty, the main eigenvectoris unique up to positive multiplicative constants and the only candidate for the representation of the priorities derived from a positive almost coherent ant symmetric matrix of pairwise comparisons.

Analytic hierarchy process (AHP) allows for inconsistency because in the formation of opinions, people are more intention inconsistency than consistency due to the inability to accurately estimate the measure values for the known scale. This fact is complicated when we deal with uncertainty (example: $a$ is preferable than $b$ twice and $b$ is preferable than $c$ three times, but a is preferable than c only five times) and ordinal detransitivize (example: a is preferable than $b$ and $b$ is preferable than $c$, but $c$ is preferable than a) [23-26].

One of the reasons of using the full matrix of pairwise comparisons is to enhance the validity of judgments about the real world. When dealing with tangible things, the matrix of pairwise comparisons can be consistent, but far from the true values. For several reasons, a minor inconsistency can be considered as a good property, and consistency can be enhanced without knowledge of the exact values as unwanted compulsion.

If, however, coherence has been achieved, people have to act like robots and can't change their opinions when receiving new information and won't be able to analyze their judgments that reflect their feelings and preferences.

Analytic hierarchy process also uses the principle of hierarchical composition to build a composite of the properties of the alternatives against multiple criteria for their properties in respect of each individual criterion. The principle consists of multiplication of each priority of (weight) alternatives by the priority (weight) of the corresponding criterion and addition according to all criteria to obtain a generalized priority of (weight) alternatives for all criteria.

The additive approach is critical and makes the composition using the limiting powers of the priorities (weights) preferable than matrix of comparisons when making decisions takes 
into account the interdependence and feedback. Various methods of constructing priorities in the same hierarchy may lead to different final values of weights of alternatives [23, 25-29].

But, despite all of the above, the question remains, what the priority is or in a more general sense, what meaning should contain a priority vector of the set of alternatives. The first option is the numerical ranking of the alternatives that indicates an order of preference among them.

The second option is that the order should also reflect intensity or cardinal preference indicated by the ratio of numerical values and, thus, the only accurate to a positive multiplicative constant (a similarity transformation). It is important to understand how it relates to the principle of hierarchical composition under a single criterion.

It is also important to consider what condition should satisfy the priority vector to remain invariant under the principle of hierarchical composition. The vector of priorities needs to reproduce itself on a relative scale, because it consists of relations that are valid preferences.

Thus, a necessary condition that must be satisfied by the vector of priorities is not only that in which it must belong to a scale of relations, which means it should remain unchanged when multiplied by a positive constant, but also it should be invariant under a hierarchical composition determined by matrix of evaluations so that new vector of priorities from that matrix won't arose. In the end, a priority vector $\mathrm{x}$ must satisfy the following relation $\mathrm{Ah}=$ $\mathrm{CX}, \mathrm{C}>0$.

Because of the need for invariance of the resulting vector of priorities, $\mathrm{x}$ must be equal to the main own vector of matrix A and c should be its own main number. The studied problem for a positive anti-symmetric matrices and their priorities is the following special case:

According to Saaty's theorem, for a given positive matrix A the only positive and the only positive constant $\mathrm{c}$ satisfy the equality $\mathrm{Ax}=\mathrm{cx}$, it is vector $\mathrm{x}$ whict is a positive multiplier vector of the Perron (the main own vector) of the matrix A. In this case the only number $\mathrm{c}$ is the number of the Perron (main own number) of matrix A [27, 28].

In accordance with the proof by Saaty, right vector of the Perron and the number of Perrone e satisfies the requirements. It is also known that the algebraic multiplicity of the number of the Perronequals to 1 (simple root), and that there is a positive left eigenvector of the matrix A (we denote егоz) corresponding to the number of the Perron.

We suppose that therearepositive vector $y$ and a (necessary positive) scalar dsuch that $\mathrm{Ay}=\mathrm{dy}$. If $\mathrm{d}$ and care not equal to each other, in view of biorthogonality [2] y is orthogonal to the vector $\mathrm{z}$, which is impossible since both vectors are positive. If $\mathrm{d}$ and $\mathrm{c}$ are equal, then $\mathrm{y}$ and $\mathrm{x}$ are dependent since $\mathrm{c}$ has algebraic multiplicity 1 , and $\mathrm{y}$ is a positive multiplier of vector $\mathrm{x}[29,30]$.

It is also true that if you start with any vector of priorities and convert it using multiplication by A any number of times, within certain limits, the product will converge to the Perron vector of the matrix A. It is known that the main eigenvector is the vector of priorities of the coherent matrix. For such matrix $\operatorname{asa}_{\mathrm{ij}}=\mathrm{w}_{\mathrm{i}} / \mathrm{w}_{\mathrm{j}}$, and $\mathrm{Aw}=\mathrm{nw}$, it follows that the vector $\mathrm{w}=\left(\mathrm{w}_{1} \ldots ., \mathrm{w}_{\mathrm{n}}\right)$., is also the main own vector $\mathrm{A}$ and its vector of priorities when the private value $\mathrm{c}=\mathrm{n}$.

A small continuous deviation $[21 ; 25]$ from the coherent matrix A shows that the resulting almost consistent matrix has its own vector of priorities as the main eigenvector, which is a small deviation from the main eigenvector of the matrix A. Thus, we assume that the matrix of judgments is obtained as a small deviation of the selected coherent matrix constructed according to the relative scale $\mathrm{w}=(\mathrm{w} 1 ; \ldots ; \mathrm{wn})$, its vector of priorities matches with the main own vector obtained as a small deviation from $w$ [22].

But, despite all, the question is what to do with mismatched positive matrix with a large misalignment. It is necessary to improve its coherence focusing its vector of priorities.

We have a consistent of order $n$ by $n$ matrix $A: A^{k}=n^{k-1} A ; A=\left(w_{i} / w_{j}\right)$.

Almost consistent matrix is a small anti-symmetric (multiplicative) variation of the coherent matrix. It is given by the product of Adamar [22,25]: 


$$
\mathrm{A}=\mathrm{WE} \text {, where } \mathrm{W}=\left(\mathrm{w}_{\mathrm{i}} / \mathrm{w}_{\mathrm{j}}\right)
$$

and

$$
E \equiv\left(e_{i j}\right) ; e_{j i}=e_{i j}^{-1}
$$

Small values $\varepsilon_{\mathrm{ij}}$ are close to one. In contrast to the additive variations of the species $\mathrm{a}_{\mathrm{ij}}+\gamma_{\mathrm{ij}}$, ant symmetric variation $\mathrm{a}_{\mathrm{ij}} \mathrm{e}_{\mathrm{ij}}, \mathrm{e}_{\mathrm{ji}}=\mathrm{e}_{\mathrm{ij}}{ }^{-1}$ is a multiplicative. It can be converted to additive variation of the coherent matrix, as follows:

$$
\begin{gathered}
\frac{w_{i}}{w_{j}}+\gamma_{i j}=\frac{w_{i}}{w_{j}} \varepsilon_{i j}, \quad \varepsilon_{i j}=1+\frac{w_{j}}{w_{i}} \gamma_{i j}, \\
\varepsilon_{j i}=\varepsilon_{i j}^{-1}=\frac{w_{j}}{w_{i}}+\gamma_{j i}=\frac{1}{1+\frac{w_{j}}{w_{i}} \gamma_{i j}} .
\end{gathered}
$$

Take a note when ant symmetric variations exists there is a confidence that $\lambda_{\max } \geq \mathrm{n}$, that helps to determine the validity of the vector $\mathrm{w}$ as the vector of priorities for almost a coherent matrix. So we have,

$$
\sum_{j=1}^{n} \varepsilon_{i j}=\sum_{j=1}^{n} a_{i j} w_{j} / w_{i}=[A w]_{i} / w_{i}=\lambda_{\max } w_{i} / w_{i}=\lambda_{\max }
$$

Calculation:

$$
n \lambda_{\max }=\sum_{i=1}^{n}\left(\sum_{j=1}^{n} \varepsilon_{i j}\right)=\sum_{i=1}^{n} \varepsilon_{i j}+\sum_{\substack{i, j=1 \\ i \neq j}}^{n}\left(\varepsilon_{i j}+\varepsilon_{j i}\right)=n+\sum_{\substack{i, j=1 \\ i \neq j}}^{n}\left(\varepsilon_{i j}+\varepsilon_{i j}^{-1}\right) \geq n+\left(n^{2}-n\right) / 2=n^{2}
$$

shows that $\lambda_{\max } \geq \mathrm{n}$. Moreover, since $\mathrm{x}+1 / \mathrm{x} \geq 2$ for all $\mathrm{x}>0$, with the achievement of equality if and only if $\mathrm{x}=1$, it is seen that $\lambda_{\max }=\mathrm{n}$ if and only if when $\varepsilon_{\mathrm{ij}}=1$, which is equivalent to the fact that all $\mathrm{a}_{\mathrm{ij}}=\mathrm{w}_{\mathrm{i}} / \mathrm{w}_{\mathrm{j}}$. The preceding arguments show that the positive anti-symmetric matrix A has $\lambda_{\max } \geq \mathrm{n}$, with the achievement of equality if and only if $\mathrm{A}$ is consistent.

But in order to improve the validity of the vector of priorities, it is necessary to convert the anti-symmetric positive matrix into an almost consistent matrix. In practice, judgments allowing one to do the comparisons, may be sufficient to bring the matrix to almost coherent. In this case it is allowed making the decision based on available information, and new knowledge of modification of the judgment should be found.

The final question is how to build $\varepsilon$ variation for the total antisymmetric matrix. By the construction the matrix of judgements has already some coherence, as it is not arbitrary antisymmetric matrix. In addition, inconsistency in the matrix can be the consequence of a mistake, such as the entry $a_{\mathrm{ji}}$ instead of $\mathrm{a}_{\mathrm{ij}}$ in position $(\mathrm{i}, \mathrm{j}$ ), with detection and correction of which the matrix can become almost coherent, or at least can improve the consistency of the matrix A.

As the main eigenvector is necessary for representing preferences (and priorities when the closeness to consistency is achieved), the algorithm based on the vector whose existence is guaranteed by Perron theory for positive matrices should be used, to improve consistency antisymmetric matrix, until almost consistency will be achieved. For a given positive antisymmetric matrix $\mathrm{A}=\left(\mathrm{a}_{\mathrm{ij}}\right)$ and a given pair of certain indexes $k>l$, let us define [22-25]

$$
\mathrm{A}(\mathrm{t})=\left[\mathrm{a}_{\mathrm{ij}}(\mathrm{t})\right] \quad \text { by } \mathrm{a}_{\mathrm{kl}}(\mathrm{t})=\mathrm{a}_{\mathrm{kl}}+\mathrm{t} ; \mathrm{a}_{\mathrm{lk}}(\mathrm{t})=\left(\mathrm{a}_{1 \mathrm{k}}+\mathrm{t}\right)^{-1}
$$


and

$$
a_{i j}(t)=a_{i j} \text { for all } i>k ; j>1 \text {, so that } A(0)=A \text {. }
$$

Let $\lambda_{\max }(t)$ denotes Peron's eigen value of the matrix $A(t)$ for all $t$ in the neighborhood of $\mathrm{t}=0$, which is small enough to be sure that all of the resulting matrices $A(t)$ are positive.

Finally, let $\mathrm{v}=\left(\mathrm{v}_{\mathrm{i}}\right)$ be the only positive own vector of positive matrix, which is normalized so that $\mathrm{v}^{\mathrm{T}} \mathrm{W}=1$. Then the classical variance formula [2, Theorem 6.3.12] shows that

$$
\left.\frac{d \lambda_{\max }(t)}{d t}\right|_{t=0}=\frac{v^{T} A^{\prime}(0) w}{v^{T} w}=v^{T} A^{\prime}(0) w=v_{k} w_{l}-\frac{1}{a_{k l}^{2}} v_{l} w_{k}
$$

Therefore,

$$
\frac{d \lambda_{\max }(t)}{d a_{i j}}=v_{i} w_{j}-a_{j i}^{2} v_{j} w_{i}, \text { for all } \mathrm{i}, \mathrm{j}=1,2, \ldots, \mathrm{n}
$$

As positive anti-symmetric matrices are used, then

$$
\frac{d \lambda_{\max }}{d a_{j i}}=-\frac{d \lambda_{\max }}{d a_{i j}} \text { for all } \mathrm{x} \mathrm{i} \text { and } \mathrm{j}
$$

\section{Conclusions}

So, to determine the input for $\mathbf{A}$, the regulation of which is in the class of antisymmetric matrices will lead to the greatest level of change values $\lambda_{\max }, n(n-1) / 2$ values of $\left\{\mathrm{v}_{\mathrm{i}} \mathrm{W}_{\mathrm{j}}\right.$ $\left.\mathrm{a}_{\mathrm{ij}}{ }^{2} \mathrm{~V}_{\mathrm{j}} \mathrm{W}_{\mathrm{i}}\right\} ; \mathrm{i}>\mathrm{j}$ should be checked, and (any) one of largest absolute value should be selected. This method is proposed for positive antisymmetric matrices by Harker (Harker) [19, 23].

It is demonstrated that if inconsistency is allowed in a positive antisymmetric matrix of paired comparisons, then major eigenvector is necessarily required to represent the priorities associated with the matrix, to ensure the inconsistency less than or equal to the desired level.

It should be noted that the practical importance of the method of paired comparisons consists in the fact that the proposed solution for analyzing and making complex decisions takes into account the dependence between the elements representing the considered problem on environmental planning and management.

Various network models are more accurate representation of the existing problems in life, having a high order of complexity. The proposed approach allows combining individual judgments into group judgments, taking into account the number of items, their weight, the importance.

\section{References}

1. A.A. Ajupov, N.G. Bagautdinova, L.V. Glukhova, M.I. Geraskin, Academy of Strategic Management Journal 15(1), 128-137 (2016)

2. A.A. Artamonova, A.A. Ajupov, Mediterranean Journal of Social Sciences 6(1), S3, 2429 (2015)

3. A.A. Rudenko, M.O. Iskoskov, D.V. Antipov, T.V. Polyakova, S.O. Zaharov, International Journal of Economics and Financial Issues 6(2), 219-224 (2016) 
4. S.A. Aristov, Simulation of economic systems (Publishing House of the Ural State Econ. University, Ekaterinburg, 2004)

5. G.L. Brodetsky, Economic-mathematical methods and models in logistics. Optimization procedures (Academy, M., 2015)

6. V.D. Geres, A.V. Colic, Transport management systems. Transportation logistics software (Yurayt, M., 2014)

7. S.Y. Daniel, Omsk Scientific Bulletin. - Omsk, 126(2), 76-80 (2015)

8. A.A. Rudenko, D.V. Antipov, M.O. Iskoskov, International Journal of Applied Engineering Research 11(5), 3211-3215 (2016)

9. D.J. McCarthy, S.M. Puffer, European Journal of International Management 7(1), 74111 (2013)

10. A.G.G. Maçada, J.C. Costa, M. Oliveira, C. Curado, International Journal of Automotive Technology \& Management 13(1), 18-35 (2013)

11. O.N. Yarygin, Simulation of the enterprise in ExtendLT (TSU Publishing House, Togliatti, 2012)

12. A.S. Hyder, D. Abraha, Baltic Journal of Management 3(3), 289-308 (2008)

13. C. Hametner, M. Kozek, S. Jakubek, C.H. Mayr, Mathematical and Computer Modeling of Dynamical Systems 20(3), 224-247 (2014)

14. A.A. Ajupov, A.A. Kurilova, D.U. Ivanov, Mediterranean Journal of Social Sciences 6(1), 40-44 (2015)

15. M.O. Iskoskov, L.A. Sosunova, Asian Social Science 11(8), 162-172 (2015)

16. T. Allen, Introduction to Engineering Statistics and Six Sigma: Statistical Quality Controland Design of Experiments and Systems (Springer, New York, 2011)

17. R.G. Askin, J.B. Goldberg, Design and Analysis of Lean Production Systems (Wiley, International student edition, New York, 2001)

18. E.T.S. Cheng, T. Choi, Innovative Quick Response Programs in Logistics and Supply Chain Management (International Handbooks on Information Systems) (Springer, New York, 2010)

19. P.T. Harker, Applied Mathematics and Computation, 217-232 (1987)

20. R.A. Horn, C.R. Johnson, Matrix Analysis (Cambridge University Press, New York, 1985)

21. P. Lancaster, The Theory of Matrices (Academic Press, New York, 1985)

22. T.L. Saaty, Proceedings of the Sixth International Symposium on the Analytic Hierarchy Process, Berne-Switzerland (2001)

23. T.L. Saaty, Decision Making with Dependence and Feedback The Analytic Network Process (RWS Publications, Pittsburgh, PA, 2001)

24. T.L. Saaty, L. Vargas, Journal of Mathematical Psychology 28, 2 (1984)

25. T.L. Saaty, G. Hu, Applied Mathematical Letters 11(4), 121-125 (1998)

26. L.G. Vargas, Applied Mathematics and Computation 12, 301-320 (1983)

27. T.L. Saaty, Fundamentals of Decision Making with the Analytic Hierarchy Process (RWS Publications, Pittsburgh, 1994)

28. P.H. Farquhar, A.R. Pratkanis, Management Science 39/10, 1214-1226 (1993)

29. M. McCord, R. Neufville, Empirical Demonstration that Expected Utility Decision Analysis is Not Operational (Reidel Publishing Company, Boston, 1983) 
30. R.L. Keeney, H. Raiffa, Decisions with Multiple Objectives: Preference and Value Tradeoffs (Wiley, New York, 1976) 